\title{
Immediate complications associated with high-flow cerebrospinal fluid egress during endoscopic endonasal skull base surgery
}

\author{
Report of 2 cases
}

\author{
Edward E. Kerr, M.D. ${ }^{1}$ Daniel M. Prevedello, M.D. ${ }^{1,2}$ Ali Jamshidi, M.D., ${ }^{1}$ \\ Leo F. Ditzel Filho, M.D., ${ }^{1}$ Bradley A. Otto, M.D., ${ }^{1,2}$ and Ricardo L. Carrau, M.D. ${ }^{1,2}$ \\ Departments of ' Neurological Surgery and ${ }^{2}$ Otolaryngology-Head and Neck Surgery, Wexner Medical \\ Center, The Ohio State University College of Medicine, Columbus, Ohio
}

\begin{abstract}
Endoscopic expanded endonasal approaches (EEAs) to the skull base are increasingly being used to address a variety of skull base pathologies. Postoperative CSF leakage from the large skull base defects has been well described as one of the most common complications of EEAs. There are reports of associated formation of delayed subdural hematoma and tension pneumocephalus from approximately 1 week to 3 months postoperatively. However, there have been no reports of immediate complications of high-volume CSF leakage from EEA skull base surgery. The authors describe two cases of EEAs in which complications related to rapid, large-volume CSF egress through the skull base surgical defect were detected in the immediate postoperative period. Preventive measures to reduce the likelihood of these immediate complications are presented.
\end{abstract}

(http://thejns.org/doi/abs/10.3171/2014.7.FOCUS14294)

KEY WORDS • cerebrospinal fluid leakage $\bullet \quad$ endoscopic endonasal approach
expanded endonasal approach
tension pneumocephalus

$\mathrm{T}$ THE endoscopic expanded endonasal approach (EEA) to the skull base is increasingly being used to address a variety of skull base pathologies..$^{12,13,21}$ Postoperative CSF leakage from the large skull base defects has been well described as one of the most common complications of EEAs. ${ }^{7,13}$ Though meningitis is the most common serious consequence of persistent CSF leakage after EEA skull base surgery, authors have reported cases of the associated formation of delayed subdural hematoma (SDH) and tension pneumocephalus from approximately 1 week ${ }^{15}$ to 3 months ${ }^{4}$ postoperatively. However, there have been no reports on immediate complications of high-volume CSF leakage from EEA skull base surgery. We present two cases of EEA skull base surgery in which complications of rapid, large-volume CSF loss from the created skull base defects were detected in the immediate postoperative period, and we propose preven-

\footnotetext{
Abbreviations used in this paper: EEA = endoscopic expanded endonasal approach; $\mathrm{EVD}=$ external ventricular drain; $\mathrm{RCH}=$ remote cerebellar hemorrhage; $\mathrm{SDH}=$ subdural hematoma; $\mathrm{SSEP}=$ somatosensory evoked potential; TFF = temporoparietal fascia flap; $\mathrm{TP}=$ tension pneumocephalus.
}

tive measures to reduce the likelihood of these immediate complications.

\section{Case Reports}

Case 1

Examination. An 80-year-old woman presented to the emergency room after waking with complete bilateral vision loss without light perception. A head CT demonstrated a large, 3-cm-diameter, sellar/suprasellar hemorrhagic pituitary adenoma (Fig. 1).

Operation. The patient underwent a transsellar and transplanum EEA for excision of the mass. A substantial amount of CSF egress was noted during the procedure, as the hemorrhagic tumor had eroded into the suprasellar subarachnoid space. Intraoperative somatosensory evoked potentials (SSEPs) demonstrated decreased responses from the left side of the body during the hemostasis portion of the procedure after gross-total tumor resection. At the end of the resection, the optic apparatus as well as the anterior circulation was preserved in the field, 


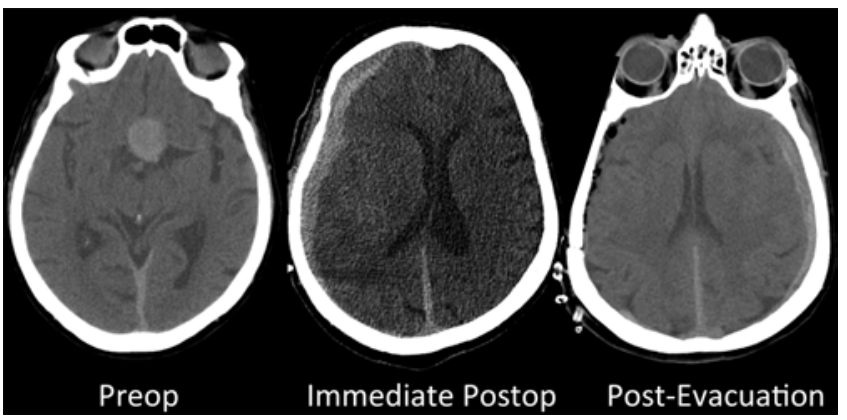

FIG. 1. Case 1. Axial CT scans demonstrating a preoperative pituitary adenoma, the immediate postoperative acute right SDH due to CSF loss, and the formation of a new contralateral SDH after evacuation of the right-sided SDH.

whereas the diaphragma sellae appeared to be destroyed by the tumor. The skull base defect was rapidly reconstructed with a pedicled nasoseptal mucoperiosteal flap, and an intraoperative CT scan of the head was obtained while the patient was still positioned in the cranial fixation pins. The scan revealed an acute right hemispheric SDH (Fig. 1). After family notification and consent and with the patient still under anesthesia, we performed an emergent right frontotemporoparietal craniotomy for SDH evacuation. During the hematoma evacuation, a frontopolar bridging vein was found to be the source of the bleeding, and it was coagulated. The brain was well relaxed after the hematoma evacuation, and we decided to reinsert the bone flap. A CT scan of the head obtained immediately after this procedure showed complete evacuation of the right SDH with an interval development in a small contralateral acute hemispheric $\mathrm{SDH}$, which we elected to monitor conservatively.

Postoperative Course. Postoperatively, the patient had no new deficits and remained cognitively intact. She did not regain light perception prior to discharge from the hospital to a rehabilitation facility 8 days later.

Case 2

Examination. A 74-year-old man presented to an outside institution with headache and diplopia. Magnetic resonance imaging of the brain revealed a large, heterogeneously enhancing, expansile mass measuring $2.3 \times 2.9 \times$ $3.5 \mathrm{~cm}$ in the suprasellar cistern as well as erosion of the clivus (Fig. 2). The patient had undergone a transsphenoidal approach elsewhere along with a biopsy of this mass, which was identified as a chordoma, so he was referred to our institution for further surgery.

Operation. We performed a repeat transsellar and transclival EEA with complete resection of the chordoma. At the end of the resection, there was an approximately 2 -mm-diameter dural opening at the clival area. The previous surgeons had performed a very large posterior nasal septectomy, eliminating the possibility of any nasoseptal mucoperiosteal flap elevation. Our alternative was to harvest a vascularized temporoparietal fascia flap (TFF), as we have described previously? ${ }^{7}$ Approximately 120 minutes were required for the TFF harvest, and dur-
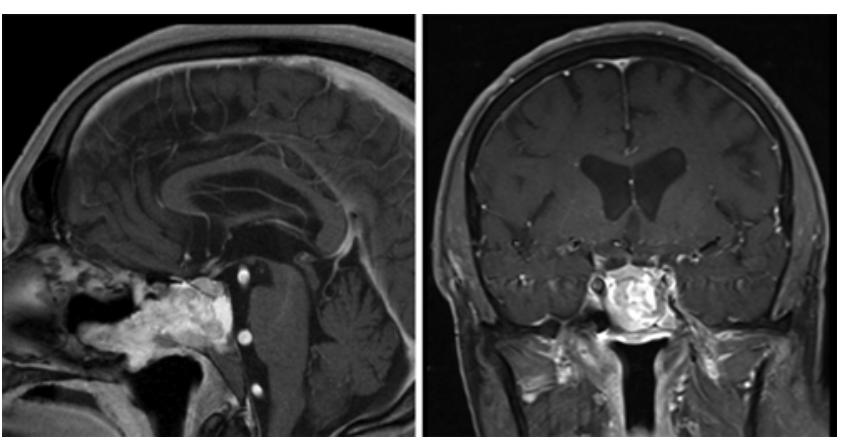

FIG. 2. Case 2. Sagittal (left) and coronal (right) MR images of the brain with contrast demonstrating a clival chordoma, for which an EEA was used for resection.

ing this time there was free CSF egress from the skull base defect, as it was left uncovered. Somatosensory evoked potentials were monitored during this case; however, they did not demonstrate any changes. Immediately postoperatively, the patient experienced a seizure and did not regain consciousness, so a head CT was obtained and demonstrated tension pneumocephalus (TP) and bilateral cerebellar hemispheric hemorrhage (Fig. 3). The intracranial air was emergently evacuated through a small twist drill bur hole (Fig. 4), the air was exchanged with saline, and a subdural drain was placed to prevent further accumulation of intracranial air under pressure.

Postoperative Course. The cerebellar hemorrhage was interpreted as not life threatening, and it was managed with serial imaging alone (Fig. 5). The patient's neurological status did not improve despite radiographic improvement, so he was clinically managed with continuous electroencephalography telemetry and external ventricular drainage in the intensive care unit. After 5 days in the coma with supportive care, the patient began to spontaneously regain consciousness. He was weaned off the external ventricular drain (EVD), which was then removed, and he was discharged to a skilled nursing facility on postoperative Day 11. He returned to our institution 1 day later with altered mental status, fever, and leukocytosis.

A CT of the head demonstrated worsening pneumocephalus and mild hydrocephalus necessitating emergent placement of an EVD. The patient was taken to the oper-
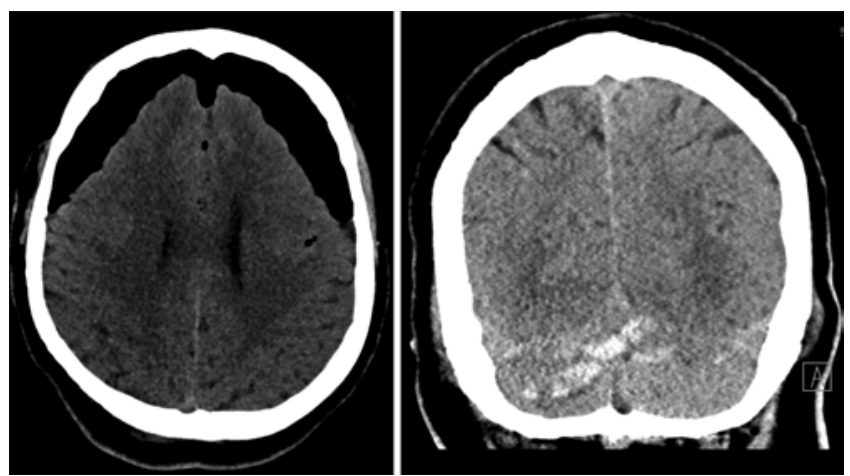

FIG. 3. Case 2. Axial (left) and coronal (right) CT demonstrating TP with a "Mount Fuji" sign and bilateral cerebellar hemispheric hemorrhage consequent to the rapid CSF loss and a prolonged open skull base defect during an EEA. 


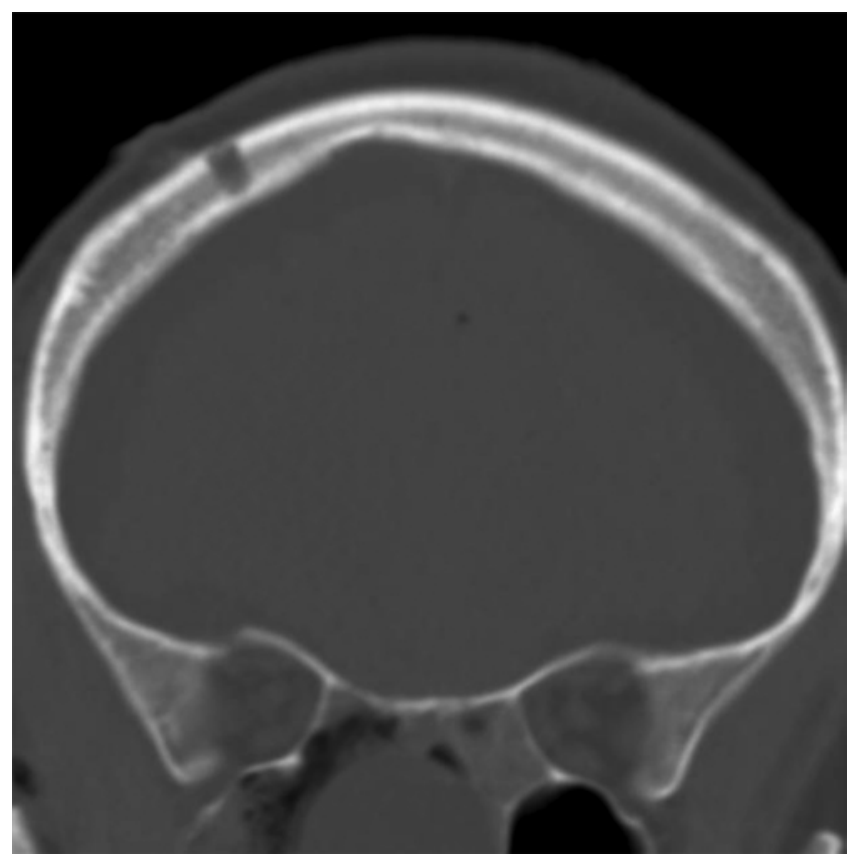

FIG. 4. Coronal bone window CT scan demonstrating the twist drill bur hole that was made emergently to drain the TP.

ating room for exploration, and a CSF leak was detected. Collagen matrix and fat graft were installed to reinforce the reconstruction, and the CSF leak was repaired. The patient's CSF study showed marked pleocytosis (558), and Klebsiella pneumoniae ultimately grew from it. Tailored antibiotic therapy commenced, he was again gradually weaned from the EVD, and he remained on intravenous antibiotics for 3 weeks. Seventeen days after his second admission, he was discharged in a debilitated state but neurologically intact from our institution to an acute inpatient rehabilitation center, from which he was ultimately discharged to his home. At the 1-year postoperative follow-up, he continued to exhibit no neurological deficit and showed no radiographic evidence of recurrence.

\section{Discussion}

Much attention has been paid to long-term CSF leak complications in skull base surgery both before and after the advent and widespread application of EEAs. . $^{3,13,18}$
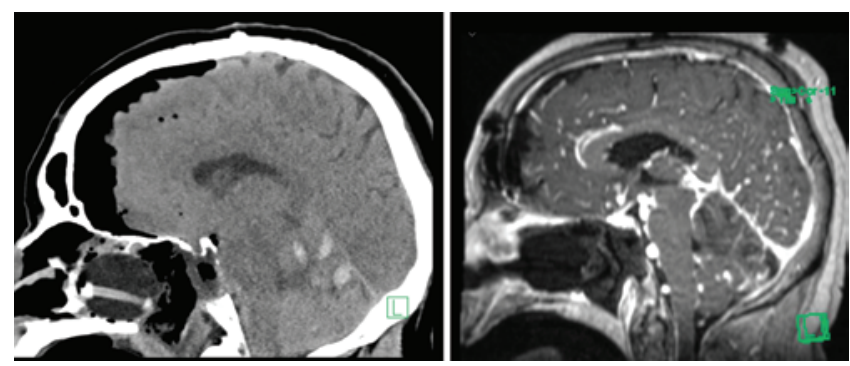

FIG. 5. Case 2. Sagittal CT scan (left) demonstrating the immediate postoperative findings of TP and cerebellar hemorrhage. Sagittal MR image (right) of the brain with contrast obtained a few hours after pneumocephalus evacuation, demonstrating total resection of the tumor and pneumocephalus improvement.
Consequently, increasingly complex multilayered repairs and ultimately vascularized pedicled mucoperiosteal flaps have been developed to reduce the risk of these longterm complications. ${ }^{10,11}$ Nevertheless, CSF leaks continue to occur at a low rate, and case reports of consequent delayed complications have been presented.,15 While the aforementioned literature focuses on complications related to low-rate CSF leakage over a protracted period, immediate complications associated with dural opening and CSF egress during an EEA have not been reported. The present report documents complications related to the immediate loss of a relatively large volume of CSF at a high rate intraoperatively.

Subdural hematoma represents one of the most potentially devastating complications of chronic CSF leakage. The mechanism by which it occurs is by inferior displacement of the cerebrum and cerebellum due to low intracranial pressure and the lack of CSF buffering, which in turn causes stretch and tearing of the cortical bridging veins and arachnoidal microvasculature, more commonly encountered in the elderly. ${ }^{1}$ In Case 1, the rapid loss of CSF from the dural defect caused the acute SDH to form over the convexity, and this was detected by SSEPs. We routinely monitor SSEPs in EEAs to ensure brain perfusion in the rare event of a carotid artery rupture as well as to certify the absence of neural or vascular compromise, permitting early assessment and intervention in the event of a change. In this case, SSEPs were particularly helpful in prompting an immediate intraoperative head CT, revealing the SDH in a timely fashion and allowing appropriate immediate treatment.

Cerebrospinal fluid loss has been similarly associated with remote cerebellar hemorrhage $(\mathrm{RCH})$, characterized by either unilateral or bilateral bleeding into the cerebellar parenchyma in the absence of direct iatrogenic injury to any posterior fossa contents during a procedure. Authors have documented RCH occurring in conjunction with supratentorial surgery, spinal surgery, and simply with lumbar drainage. ${ }^{8,9,16}$ Though the mechanism by which $\mathrm{RCH}$ occurs has not been demonstrated experimentally, most authors agree that it is attributable to venous infarction. The hypothesis holds that rapid loss of a large volume of CSF leads to caudal displacement of the cerebellum, which stretches draining veins to the point of occlusion, which then causes venous congestion and ultimately parenchymal hemorrhage..$^{8,9,16}$ These veins can also rupture and cause local subarachnoid hemorrhage, which is commonly seen in association with $\mathrm{RCH}$. Case 2 provides a clear example of not only this potentially devastating complication but also TP, which was a concomitant but unrelated complication of CSF egress.

Tension pneumocephalus, an accumulation of intracranial air under pressure, has been described as a consequence of trauma, skull base erosion by neoplasm, lumbar puncture, posterior fossa surgery in the sitting position, and endoscopic sinus surgery. ${ }^{14,19}$ As in $\mathrm{RCH}$, no clinical experimental model exists to explain the phenomenon of TP, but two hypotheses exist, both related to dural violation and CSF displacement. The first is the "inverted bottle" hypothesis, in which CSF loss through a dural defect generates a negative intracranial pressure relative to 
the atmosphere, which causes air to rush into the subdural space along the pressure gradient and collect in the superior-most area. The second is the "ball valve" hypothesis, in which a dural defect is shaped so that it allows air to enter the subdural space but coapts when the intradural pressure rises beyond a certain point, trapping the air in the subdural space. ${ }^{14,19}$ We believe that both of these hypotheses are plausible and not mutually exclusive. Skull base surgeries certainly create defects by which the inverted bottle hypothesis could lead to TP. At the conclusion of surgery, some form of inlay or onlay graft is used to close the defect, whose free edges may act as the ball valve that permits the entry of nasopharyngeal air into the subdural space while preventing its egress. In Case 2, these mechanisms most likely occurred during the flap harvesting since the clival dural defect was left uncovered. Of note, we did not use a lumbar drain in either of our cases, and their routine use in skull base surgery has proved controversial. Lumbar drainage has been advocated by cranial base surgeons to divert CSF to minimize the force required for frontal lobe retraction intraoperatively as well as to potentially decrease CSF leak rates postoperatively with prolonged usage by minimizing CSF egress through the defect, allowing it to heal., ${ }^{2,5,6,17}$ However, the literature also cites numerous instances of TP complicating skull base surgery in which continuous lumbar drainage was used. ${ }^{17,20}$ After a skull base defect is created, continuous lumbar drainage may promote the ingress of air through the skull base dural defect by creating a relatively lower pressure than the atmospheric pressure in the subarachnoid space. ${ }^{14}$ Consequently, we reserve lumbar drain use for select cases of refractory CSF leakage, with close monitoring for the development of TP.

To prevent neurological injury related to TP, $\mathrm{RCH}$, and $\mathrm{SDH}$, we recommend preventive measures aimed at minimizing CSF egress, preventing entry of intracranial air, and vigilant monitoring for these complications to facilitate timely management. Endoscopic expanded endonasal approaches for skull base pathology often require significant operative time to achieve the surgical goals. To minimize the risk of entraining air during these oftenlengthy procedures, we recommend returning the bed flat during the intradural portion of the surgery, since the head is frequently elevated to decrease venous pressure and bleeding during the extradural portion of the procedure. The greater the difference in height between any subdural portion of the neuraxis and the dural defect, the greater the chance of experiencing the "inverted bottle" phenomenon and risking TP development. Moreover, when turning attention to other surgical matters after creating a dural defect, as occurred during our TFF harvest, we recommend impeding CSF egress with either a cottonoid patty or a temporarily inlaid collagen matrix. Any maneuver to decrease CSF egress during intradural dissection, by covering part of the skull base defect or adding patties in the subarachnoid space, is also advocated, particularly when performing surgery in elderly patients. As regards monitoring, we advocate SSEP monitoring for all EEAs not only for the rare event of a vascular injury, but also for the provision of intraoperative evidence of the accumulation of subdural air or blood. Finally, we recom- mend an intraoperative or immediate postoperative head CT for all patients undergoing an EEA with dural entry to monitor for, and to guide the clinical management of, these potential complications.

\section{Conclusions}

Quite appropriately, the bulk of the literature concerned with the complications of EEA has focused on the long-term ones, as they clearly occur more often than the immediate complications related to dural entry. However, the cases presented here highlight the importance of recognizing and addressing these immediate complications. Based on our experience, we believe that patients undergoing prolonged EEA skull base surgery may be best managed with intraoperative maneuvers to impede CSF loss and air entry, as well as with intraoperative neurological monitoring and immediate postoperative imaging.

\section{Disclosure}

The authors report no conflict of interest concerning the materials or methods used in this study or the findings specified in this paper.

Author contributions to the study and manuscript preparation include the following. Conception and design: Prevedello. Acquisition of data: Prevedello, Jamshidi, Ditzel Filho, Otto, Carrau. Analysis and interpretation of data: all authors. Drafting the article: Kerr. Critically revising the article: Prevedello, Kerr. Reviewed submitted version of manuscript: all authors. Approved the final version of the manuscript on behalf of all authors: Prevedello.

\section{References}

1. Acharya R, Chhabra SS, Ratra M, Sehgal AD: Cranial subdural haematoma after spinal anaesthesia. Br J Anaesth 86: 893-895, 2001

2. Bien AG, Bowdino B, Moore G, Leibrock L: Utilization of preoperative cerebrospinal fluid drain in skull base surgery. Skull Base 17:133-139, 2007

3. Blount A, Riley K, Cure J, Woodworth BA: Cerebrospinal fluid volume replacement following large endoscopic anterior cranial base resection. Int Forum Allergy Rhinol 2:217-221, 2012

4. Dallan I, Lenzi R, Muscatello L, Bignami M, Battaglia P, Castelnuovo P: Subdural haematoma after endoscopic skull base surgery: case report and lesson learned. Clin Neurol Neurosurg 113:496-498, 2011

5. Dias FL, Sá GM, Kligerman J, Lopes HF, Wance JR, Paiva FP, et al: Complications of anterior craniofacial resection. Head Neck 21:12-20, 1999

6. Donald PJ: Complications in skull base surgery for malignancy. Laryngoscope 109:1959-1966, 1999

7. Fortes FS, Carrau RL, Snyderman CH, Kassam A, Prevedello D, Vescan A, et al: Transpterygoid transposition of a temporoparietal fascia flap: a new method for skull base reconstruction after endoscopic expanded endonasal approaches. Laryngoscope 117:970-976, 2007

8. Friedman JA, Ecker RD, Piepgras DG, Duke DA: Cerebellar hemorrhage after spinal surgery: report of two cases and literature review. Neurosurgery 50:1361-1364, 2002

9. Friedman JA, Piepgras DG, Duke DA, McClelland RL, Bechtle PS, Maher CO, et al: Remote cerebellar hemorrhage after supratentorial surgery. Neurosurgery 49:1327-1340, 2001

10. Hadad G, Bassagasteguy L, Carrau RL, Mataza JC, Kassam A, Snyderman $\mathrm{CH}$, et al: A novel reconstructive technique 


\section{Immediate complications of cerebrospinal fluid loss}

after endoscopic expanded endonasal approaches: vascular pedicle nasoseptal flap. Laryngoscope 116:1882-1886, 2006

11. Kassam A, Carrau RL, Snyderman CH, Gardner P, Mintz A: Evolution of reconstructive techniques following endoscopic expanded endonasal approaches. Neurosurg Focus 19(1):E8, 2005

12. Kassam A, Snyderman CH, Mintz A, Gardner P, Carrau RL: Expanded endonasal approach: the rostrocaudal axis. Part I. Crista galli to the sella turcica. Neurosurg Focus 19(1):E3, 2005

13. Kassam A, Snyderman CH, Mintz A, Gardner P, Carrau RL: Expanded endonasal approach: the rostrocaudal axis. Part II. Posterior clinoids to the foramen magnum. Neurosurg Focus 19(1):E4, 2005

14. Lunsford LD, Maroon JC, Sheptak PE, Albin MS: Subdural tension pneumocephalus. Report of two cases. J Neurosurg 50:525-527, 1979

15. Mammis A, Agarwal N, Eloy JA, Liu JK: Intraventricular tension pneumocephalus after endoscopic skull base surgery. J Neurol Surg A Cent Eur Neurosurg 74 Suppl 1:e96-e99, 2013

16. Nam TK, Park SW, Min BK, Hwang SN: Remote cerebellar hemorrhage after lumbar spinal surgery. J Korean Neurosurg Soc 46:501-504, 2009

17. Pepper JP, Lin EM, Sullivan SE, Marentette LJ: Perioperative lumbar drain placement: an independent predictor of tension pneumocephalus and intracranial complications following anterior skull base surgery. Laryngoscope 121:468-473, 2011
18. Schwaber MK, Netterville JL, Coniglio JU: Complications of skull base surgery. Ear Nose Throat J 70:648-654, 659-660, 1991

19. Simmons J, Luks AM: Tension pneumocephalus: an uncommon cause of altered mental status. J Emerg Med 44:340-343, 2013

20. Yates H, Hamill M, Borel CO, Toung TJ: Incidence and perioperative management of tension pneumocephalus following craniofacial resection. J Neurosurg Anesthesiol 6:15-20, 1994

21. Zada G, Du R, Laws ER Jr: Defining the "edge of the envelope": patient selection in treating complex sellar-based neoplasms via transsphenoidal versus open craniotomy. Clinical article. J Neurosurg 114:286-300, 2011

Manuscript submitted June 9, 2014.

Accepted July 21, 2014.

Portions of this work were presented in poster form at the 24th Annual North American Skull Base Society Meeting held in San Diego, California, on February 14, 2014.

Please include this information when citing this paper: DOI: 10.3171/2014.7.FOCUS14294.

Address correspondence to: Daniel M. Prevedello, M.D., Department of Neurological Surgery, The Ohio State University Wexner Medical Center, 410 W. 10th Ave., N-1049 Doan Hall, Columbus, OH 43210. email: daniel.prevedello@osumc.edu. 